\title{
Comparison of virulence factors and antibiotic resistance of Pseudomonas aeruginosa strains isolated from patients with and without cystic fibrosis
}

\author{
Salih Macin ${ }^{1 *}$, Meral Akarca², Burcin Sener ${ }^{3}$, Yakut Akyon ${ }^{3}$ \\ 1. Selcuk University Faculty of Medicine, Department of Medical Microbiology, Konya, Turkey \\ 2. Golbasi State Hospital, Department of Medical Microbiology, Ankara, Turkey \\ 3. Hacettepe University Faculty of Medicine, Department of Medical Microbiology, Ankara, Turkey.
}

\begin{abstract}
Its rising incidence, virulence factors and antibiotic resistance rate makes it difficult to treat Pseudomonas aeruginosa infections. The aim of this study was to compare virulence factors and antibiotic resistance of P. aeruginosa isolates from cystic fibrosis (CF) and other lower respiratory tract infections.

Isolates from patients $(n=125)$ were divided into two groups. The isolates in the first group were from CF patients ( $n=64)$. And in the other group isolates were from lower respiratory tract samples, from patients that did not have $C F(n=61)$. The antibiotic susceptibility tests were done by using disc diffusion method. As phenotypic tests; DNase, protease, elastase, hemolysis, and motility test were performed.

The mucoid form of $P$. aeruginosa was detected in 29.7\% of CF patients' isolates, whereas in the other group (non-CF) this rate was $9.8 \%(p=0.011)$. Motility in the CF patients' isolates was lower $(84.4 \%)$ then the other group (96.7\%). The presence of DNase was significantly low in CF patients' isolates when compared to the other group $(p=0.009)$. When the antibiotic resistance was compared; ceftazidime, imipenem and meropenem and piperacillin resistance was found significantly low in CF patients compared to isolates from the other group $(p \leq 0.05)$.

Information about virulence factor patterns and antibiotic resistance of P. aeruginosa isolates from patients with cystic fibrosis and the patients without cystic fibrosis can prevent the unnecessary usage of antibiotics and lead the way to new approaches in treatment.
\end{abstract}

Keywords: Antibiotic susceptibility, Cystic fibrosis, Pseudomonas aeruginosa, virulence

Received: 22 ${ }^{\text {nd }}$ March 2017; Accepted: 20 $0^{\text {th }}$ July 2017; Published: $18^{\text {th }}$ August 2017

\section{Introduction}

Pseudomonas aeruginosa which is an opportunistic Gram-negative bacillus, can easily be isolated from the environmental sources and even antiseptic solutions due to its hydrophilic nature while it rarely colonizes healthy people (1). P. aeruginosa regulates its pathogenicity through a series of virulence factors such as adhesins, pyocyanin, elastase, proteases, hemoly-

*Corresponding author: Salih Maçin, Sirnak State Hospital, Microbiology Laboratory, 73000 Şırnak, Turkey, e-mail: salihmacin@hotmail.com 
sins, exotoxin and exoenzyme S (2). P. aerugino$s a$ exhibit high adaptation ability to enormously different settings owing to its varying potential of virulence factors plus its intrinsic resistance to several antibiotics. Inducible chromosomal AmpC beta - lactamases and efflux pumps systems are among the most frequent mechanisms of intrinsic resistance in $P$. aeruginosa isolates $(3,4)$. The treatment of P.aeruginosa infections is challenging since acquired resistance can develop against broad-spectrum penicillins, extended-spectrum cephalosporins, monobactams, aminoglycosides and colistin which are applied as treatment alternatives.

$P$. aeruginosa is the most frequently isolated pathogen from the respiratory tract of patients with cystic fibrosis (CF) (5). Colonization and infection of the respiratory tract with $P$. aeruginosa is persistent in the CF setting. During the chronic colonization/infection process P.aeruginosa undergoes some genotypic and phenotypic changes such as mucoid colony formation, loss of motility and hyperexpression of its virulence factor or resistance genes (6). These alterations enable the bacteria to adapt to the stressful environment in $\mathrm{CF}$ airways.

It is well-known that P.aeruginosa is also a frequent cause of acute nosocomial infections such as bloodstream infections, urinary tract infections, pneumonia or wound infections. Regulation and expression of virulence factors of bacteria are different in acute and chronic infection settings (7). This study was aimed to compare the virulence factors and antibiotic susceptibilities of $P$. aeruginosa strains that isolated from patients with and without $\mathrm{CF}$.

\section{Materials and methods}

\section{Pseudomonas aeruginosa isolates}

$P$. aeruginosa isolates obtained from $64 \mathrm{CF}$ patients and 61 non-CF patients with lower respiratory tract infection were included in the study. The isolates were collected between June 2013 and December 2013 and the study was performed between January 2014 and June 2014. Only one isolate from each patient was included into the study. Oxidase positive, Gram negative non-glucose fermenting bacteria with pigment formation were presumably identified as $P$. aeruginosa and the identification was confirmed with Phoenix Automated Bacterial Identification System (Becton Dickinson, USA). P. aeruginosa PAO1, PAO - JP2 and PAO - JP3 were used as the control strains. PAO1 is the wild type strain, PAO-JP2 and PAO-JP3 are the quorum sensing lasI and rhlI mutants and they are negative controls for elastase and protease activity.

\section{Antibiotic Susceptibility Tests}

The antibiotic susceptibilities of the P. aeruginosa strains against amikacin, gentamicin, tobramycin, ceftazidime, cefepime, imipenem, meropenem, ciprofloxacin, levofloxacin, piperacillin and colistin was determined by Kirby - Bauer disk diffusion method according to CLSI criteria (8). P. aeruginosa ATCC 27853 was used as the standard control strain for susceptibility testing.

\section{Phenotypic determination of the virulence factors}

Elastase activity: Elastin Congo red (ECR) measurement method was used to investigate elastase activity (9). All clinical strains, $P$. aeruginosa PAO1, PAO - JP2 and PAO - JP3, were incubated at $37^{\circ} \mathrm{C}$ overnight. In order to determine the semi-quantitative elastase production, the centrifugation was done for the prepared suspensions of the clinical $P$. aeruginosa strains and PAO1, PAO - JP2 and PAO - JP3 strains. $1 \mathrm{ml}$ of $30 \mathrm{mM}$ Tris and $10 \mathrm{mg}$ of elastin - Congo red ( $\mathrm{pH}$ 7.2) were added into tubes containing 0.5 $\mathrm{ml}$ of the bacterial supernatants. The tubes were incubated on a shaker at $37^{\circ} \mathrm{C}$ for 24 hours, followed by centrifugation at $3000 \mathrm{x} \mathrm{g}$ for 10 
minutes. From each tube $200 \mathrm{~mL}$ of the supernatant was removed and placed into two wells of a microtiter plate and the absorbance was measured at $495 \mathrm{~nm}$ with an optical reader. For each isolate, the average value of the two wells was calculated. In order to determine the cut-off value, two standard deviations were added to the average value from the study with the PAO - JP2 and PAO - JP3 strains which are negative for elastase production. The cut-off value was determined as 0.474 with $95 \%$ confidence interval and the isolates with the absorbance value > 0.474 at $495 \mathrm{~nm}$ was evaluated as positive for elastase activity.

Alkaline protease activity: For the determination of alkaline protease activity, skim milk agar (SMA) was used. $20 \mu \mathrm{l}$ of the bacterial supernatant was placed with sterile Pasteur pipette into the wells opened in SMA. The medium was incubated at $25^{\circ} \mathrm{C}$ for $18-24$ hours. The presence of a transparent zone around the wells with the bacteria was interpreted as positive for protease activity (10).

Hemolysis activity: The strains were inoculated into 5\% sheep blood agar and after the incubation at $37^{\circ} \mathrm{C}$ for 24 hours, production of hemolysis was determined.

Motility test: The strains were stabbed into the semi-solid motility test medium with an inoculation loop. Cultures were incubated at $37^{\circ}$ C for 24 - 48 hours. The extension from the stab line and production of turbidity or cloudiness throughout the medium was regarded as a positive motility test. P. aeruginosa ATCC 27853 strain was used as the positive control.

DNAse activity: The bacteria grown in blood agar were inoculated by drawing a line onto DNase agar (Oxoid, UK). Plates were incubated at $37^{\circ} \mathrm{C}$ aerobically for 18 to 24 hours. Lack of clearance around bacterial colonies was considered as DNase negative. The generation of clear zones around inoculation line was considered as DNase positive.
Mucoid colony morphology: All clinical samples were inoculated onto Mueller Hinton agar and incubated for 24 hours at $37^{\circ} \mathrm{C}$ for the detection of mucoid colony formation.

Limitation of the study is not to perform virulence gene associated genes.

\section{Statistical evaluation}

The difference between groups from categorical variables was studied using Pearson Chi Square test, Yates adjusted Chi - square and Fisher's exact chi - square test. In the study, p-value lower than 0.05 was considered as statistically significant. All analysis were performed with the help of IBM SPSS (Statistical Package for Social Science) Statistics 21.0 software.

\section{Results}

The mean age of the patients with $\mathrm{CF}$ was $13.63 \pm 1.05$ years while it was $44.57 \pm 3.21$ for non-CF patients. A total of 125 P.aeruginosa isolates were included in this study, 64 of them were from sputum samples of patients with $\mathrm{CF}$ while 61 were isolated from lower respiratory tract samples of non-CF patients (28 sputum (45.2\%), five bronchoalveolar lavage (8.2\%), 28 tracheal aspirate samples $(45.3 \%))$.

While presence of elastase and protease was $84.4 \%$ and $70.3 \%$, respectively in CF isolates, these rates were $70.5 \%$ and $62.3 \%$, respectively, for non-CF isolates $(\mathrm{p}=1.0, \mathrm{p}=0.448)$ The rate of motility was higher in non-CF isolates $(96.7 \%)$ than the CF isolates (84.4\%) and the difference was statistically significant $(\mathrm{p}=0.042)$. Mucoid colony morphology was detected in a significantly high rate of CF isolates $(29.7 \%)$ than the nonCF isolates $(9.8 \%),(p=0.011)$. DNase activity was found to be statistically significantly lower for $\mathrm{CF}$ isolates than non-CF isolates $(\mathrm{p}=0.009)$. Regarding the presence of hemolysis, no statistically significant differences were found between the two groups (Table 1). 
When the change in virulence factors according to the age of $\mathrm{CF}$ patients examined; the presence of DNase was detected in $27.2 \%$ of patients older than 18 years and only in $9.5 \%$ of patients under 18 ages. Consistent with this result, DNase positivity was found to be $45.1 \%$ in adults in the non-CF group while there was no DNase positivity in pediatric group. No significant differences were found in other virulence factors (Table 2).

The antibiotic susceptibility data revealed that the rates of ceftazidime, imipenem, meropenem and piperacillin resistance were lower in $\mathrm{CF}$ isolates than the non-CF isolates $(\mathrm{p} \leq 0.05)$. Colistin resistance was not determined in any of the $P$. aeruginosa strains tested (Table 3 ).

\section{Discussions}

$P$. aeruginosa which exhibits a wide range of virulence factors causes a diversity of infections with high mortality and morbidity. These opportunistic pathogens are widespread microorganisms in nature. Although they do not cause disease in healthy individuals, it may lead to life-threatening infections in patients with underlying disease such as cystic fibrosis, malignancies, burns, immunosuppressive and traumatic injuries (11).

Phenotypic analysis of the isolates revealed that mucoid colony formation was $29.7 \%$ in $\mathrm{CF}$ isolates and this was found to be statistically significantly higher than the non-CF group $(9.8 \%)$ $(\mathrm{p}=0.011)$. This finding supported the view that

Table 1. The comparison of virulence factors of Pseudomonas aeruginosa isolates from patients with and without cystic fibrosis.

\begin{tabular}{lccccc}
\hline \multirow{2}{*}{ Virulence factor } & \multicolumn{2}{c}{$\mathrm{CF}$ isolates $(\mathrm{n}=64)$} & \multicolumn{2}{c}{ Non-CF isolates $(\mathrm{n}=61)$} & \multirow{2}{*}{$\mathrm{p}$-value } \\
\cline { 2 - 5 } & $\mathrm{n}$ & $\%$ & $\mathrm{~N}$ & $\%$ & \\
\hline Elastase & 54 & 84.4 & 43 & 70.5 & 1.0 \\
\hline Protease & 45 & 70.3 & 38 & 62.3 & 0.448 \\
\hline Motility & 54 & 84.4 & 59 & 96.7 & 0.042 \\
\hline Hemolysis & 47 & 73.4 & 51 & 83.6 & 0.245 \\
\hline DNase & 10 & 15.6 & 23 & 37.7 & 0.009 \\
\hline Mucoid colony & 19 & 29.7 & 6 & 9.8 & 0.011 \\
\hline
\end{tabular}

Table 2. The comparison of virulence factors of Pseudomonas aeruginosa isolates from pediatric patients and adult patients

\begin{tabular}{lcccccc}
\hline & \multicolumn{2}{c}{ CF isolates $(\mathrm{n}=64)$} & \multicolumn{4}{c}{ Non-CF isolates $(\mathrm{n}=61)$} \\
\cline { 2 - 7 } Virulence factor & $\begin{array}{c}<18 \text { age } \\
(\mathrm{n}=42) \\
\mathrm{n}(\%)\end{array}$ & $\begin{array}{c}>18 \text { age } \\
(\mathrm{n}=22) \\
\mathrm{n}(\%)\end{array}$ & p-value & $\begin{array}{c}<18 \text { age } \\
(\mathrm{n}=10) \\
\mathrm{n}(\%)\end{array}$ & $\begin{array}{c}>18 \text { age }(\mathrm{n}=51) \\
\mathrm{n}(\%)\end{array}$ & p-value \\
\hline Elastase & $36(85.7)$ & $18(81.8)$ & 0.72 & $8(80)$ & $35(68.6)$ & 0.70 \\
\hline Protease & $30(71.4)$ & $15(68.2)$ & 0.78 & $6(60)$ & $32(62.7)$ & 1.00 \\
\hline Motility & $36(85.7)$ & $18(81.8)$ & 0.72 & $9(90)$ & $50(98)$ & 0.30 \\
\hline Hemolysis & $33(78.6)$ & $14(63.6)$ & 0.19 & $9(90)$ & $42(82.3)$ & 0.55 \\
\hline DNase & $4(9.5)$ & $6(27.2)$ & 0.08 & 0 & $23(45.1)$ & 0.009 \\
\hline Mucoid colony & $12(28.5)$ & $7(31.8)$ & 0.78 & $1(10)$ & $5(9.8)$ & 0.98 \\
\hline
\end{tabular}


Table 3. The comparison of antibiotic resistance rate of Pseudomonas aeruginosa isolates from patients with and without cystic fibrosis

\begin{tabular}{lccccc}
\hline \multirow{2}{*}{ Antibiotics } & \multicolumn{2}{c}{ CF isolates } & \multicolumn{2}{c}{ Non-CF iso- } \\
& \multicolumn{2}{c}{$(\mathrm{n}=64)$} & \multicolumn{2}{c}{ lates (n=61) } & p-value \\
& (n) & $\%$ & $(\mathrm{n})$ & $\%$ & \\
\hline Piperacillin & 10 & 15.6 & 24 & 39.3 & 0.003 \\
\hline Ceftazidime & 4 & 6.3 & 11 & 18 & 0.040 \\
\hline Cefepime & 8 & 12.5 & 13 & 21.3 & 0.180 \\
\hline Imipenem & 11 & 17.2. & 31 & 50.8 & 0.001 \\
\hline Meropenem & 10 & 15.6 & 27 & 44.3. & 0.001 \\
\hline Amikacin & 19 & 29.7 & 18 & 29.5 & 0.980 \\
\hline Gentamicin & 17 & 26.6 & 22 & 36.1. & 0.250 \\
\hline Tobramycin & 9 & 14.1 & 13 & 21.3 & 0.280 \\
\hline Ciprofloxacin & 13 & 20.3 & 16 & 26.2 & 0.430 \\
\hline Levofloxacin & 18 & 28.1 & 17 & 27.9 & 0.970 \\
\hline Colistin & 0 & 0 & 0 & 0 & - \\
\hline
\end{tabular}

the emergence of mucoid colonies caused by overproduction of the polysaccharide alginate, which is widely considered to be a marker for the transition to chronic $P$. aeruginosa infection in CF. Thus the microenvironment in CF lung induced the formation of mucoid colonies. Besides mucoid colony formation loss of motility is also a common indicator of adaptation (6). In our study motility feature of isolates from patients with CF was (84.4\%) less than that detected in non-CF patients $(96.7 \%)$ and the difference was statistically significant $(\mathrm{p}=0.042)$. This indicated that the continous and selective pressure in the $\mathrm{CF}$ environment leads to some phenotypic changes in bacteria to help its adaptation to the host. Non-motile P.aeruginosa strains are hardly phagocytosed by macrophages and neutrophils and thus evade from the host immune system leading to chronicity.

The presence of DNase is frequent particularly in the case of severe $P$. aeruginosa infections. In our study DNase positivity for non-CF group was $37.7 \%$ whereas it was $15.6 \%$ in $\mathrm{CF}$ patients $(p=0.009)$. Extracellular DNA in the biofilm matrix could take part in the development of bacterial communities. Since $P$. aeruginosa strains isolated from $\mathrm{CF}$ patients have less DNase production, $P$. aeruginosa is unable to disrupt the alginate, which supports biofilm formation. DNase, given as a treatment in $\mathrm{CF}$ patients, reduces the mucoid extracellular matrix, making it easier for antibiotics to reach bacterial targets (12).

The relationship between $P$. aeruginosa virulence factors and the body parts bacteria isolated was examined in various studies which tried to clarify the pathogenesis of $P$. aeruginosa infections. In their study, Ciragil et al. studied elastase, protease and alginate properties of $P$. aeruginosa isolates isolated from different parts of the body. They found that alkaline protease was detected in $52 \%$ of $\mathrm{CF}$ and $65 \%$ of non-CF patients (13). In our study protease positivity was $64 \%$ for the $\mathrm{CF}$ isolates and $80 \%$ for the non-CF isolates. This supports the view that protease activity is seen more in acute P.aeruginosa infections and it decreases when the infection becomes chronic.

The importance of elastase production in the pathogenesis of lung infections due to $P$. aeruginosa was demonstrated clearly and shown that the $73 \%-95 \%$ of $P$. aeruginosa isolates from respiratory tract samples produce this enzyme (14-15). In isolates from chronic CF patients, the production of elastase is reduced (16). In our study there was no significant difference between $\mathrm{CF}$ and non-CF group ( $\mathrm{p} \geq 0.05$ ). This may be, because of the younger ages in the $\mathrm{CF}$ group. A study by Amitani et al. revealed that elastase produced by P.aeruginosa leads to delay in mucociliary clearance in patients with chronic bronchial infection (17). Also in our study, elastase positivity was found as $84 \%$ for the $P$. aeruginosa isolates from the patients with $\mathrm{CF}$.

Faraji et al. compared the prevalence of three virulent genes in $P$. aeruginosa be- 
tween CF patients' isolates and the burn wound isolates. They found that the number of tox $A, \operatorname{las} B$ and exo $S$ were significantly higher in the bacteria from the patients with CF (18). In our study we performed phenotypic tests and found high rates of elastase, protease and mucoid formation in $\mathrm{CF}$ patients. It is clear that virulence factors play an important role in $\mathrm{CF}$ infections by $P$. aeruginosa.

The CF $P$. aeruginosa isolates in this study population revealed lower beta-lactam resistance than their non-CF counterparts. In our study resistance rates of ceftazidime, imipenem, meropenem and piperacillin antibiotics were lower in $\mathrm{CF}$ isolates than the non-CF isolates $(\mathrm{p} \leq 0.05)$. Intrinsic and acquired antibiotic resistance also aid to the virulence and adaptation of P.aeruginosa in the infection setting (19). This might be explained by the higher mucoidity thus biofilm production of these more susceptible isolates. It has been previously shown that mucoid P. aeruginosa isolates, that characterize the chronic lung infection in $\mathrm{CF}$ patients, are more susceptible to antibiotics since they produce less beta-lactamase than the non-mucoid paired isolates. The authors proposed that these mucoid isolates are exposed to a relatively lower antibiotic pressure and thus produce less amount of beta-lactamase (20).

In our study we did not detect any colistin resistance while ceftazidime resistance was $6.3 \%$. Consistent with our findings in a study that aims to determine antibiotic resistance of $\mathrm{CF}$ in $P$. aeruginosa isolates, ceftazidime resistance was detected as $14 \%$ while there is no resistance for colistin (21). While previous study was detected resistance for imipenem, meropenem piperacillin and tobramycin as $20 \%, 21.4 \%, 23.2$ and $23.5 \%$ respectively; we detected resistance for these antibiotics as $17.2 \%, 15.6 \%, 15.6 \%$ and $14.1 \%$ respectively. In their study Manno et al. detected amikacin resistance as $30.2 \%$, similar to our study showing $29.7 \%$ resistance (21).
Both studies also found high resistance for amikacin. In a multicentre study in Australia, they also found that resistance for aminoglycoside as $43 \%$ in the pediatric patient group and $53 \%$ in adult patient group (22).

Bosso et al. compared the antibiotic resistance of P.aeruginosa isolates obtained from patients with CF with other groups and found that resistance rate for amikacin, gentamicin, tobramycin and levofloxacin was lower in patients with CF (23). Similar results were also obtained in another Turkish multi-center study comparing the antibiotic resistance of P.aeruginosa isolates from sputum samples of patients with $\mathrm{CF}$ and lower respiratory tract samples of patients without CF (24). In contrary to this Rao et al. showed that isolates from blood samples of CF patients were more resistant to antibiotics than those of non-CF patients (25). It may be because of several classes of antibiotics used in the management of $\mathrm{CF}$ airways infection. These data also illustrate the complexity of antibiotic susceptibility of $P$. aeruginosa within $\mathrm{CF}$.

The results of this study supported the view that progressive evolution of P.aeruginosa strains in CF patients may lead to the development of a less virulent phenotype in terms of expression of specific virulence determinants. However, mucoid colony conversion seems to be the major determinant in the chronic colonization/infection process. While $P$. aeruginosa isolates from CF patients lose their ability to provoke acute infections, they gain the ability to persist in the form of biofilm nature. These findings emphasize the need for further studies on the virulence factor and antibiotic resistance patterns of $\mathrm{CF}$ isolates in order to establish improved treatment strategies in CF lung infections.

\section{Conflict of interest}

This research did not receive any specific grant from funding agencies in the public, commercial, or not-for-profit sectors. 
This study follows the principles of the Declaration of Helsinki.

\section{Abbreviations}

CF: Cystic fibrosis

ECR: Elastin Congo red

DNAse: Deoxyribonuclease

SMA: Skim milk agar

\section{References}

1. Speert DP, Campbell ME, Davidson AG, Wong LT. Pseudomonas aeruginosa colonization of the gastrointestinal tract in patients with cystic fibrosis. J Infect Dis. 1993 Jan;167(1):226-9. DOI: 10.1093/infdis/167.1.226

2. Van Delden C, Iglewski BH. Cell-to-cell signaling and Pseudomonas aeruginosa infections. Emerg Infect Dis. 1998 Oct-Dec;4(4):551-60. DOI: 10.3201/ eid0404.980405

3. Livermore DM. Multiple mechanisms of antimicrobial resistance in Pseudomonas aeruginosa: Our worst nightmare? Clin Infect Dis. 2002 Mar;34(5):63440. DOI: $10.1086 / 338782$

4. Matroş L, Krausz TL, Pandrea SL, Ciontea MI, Chiorean E, Pepelea LS, et al. Phenotypic and genotypic study of carbapenem-resistant Pseudomonas aeruginosa strains isolated from hospitalized patients. Rev Romana Med Lab. 2016;24(2):201-11. DOI:10.1515/ rrlm-2016-0021

5. Govan JR, Deretic V. Microbial pathogenesis in cystic fibrosis: mucoid Pseudomonas aeruginosa and Burkholderia cepacia. Microbiol Rev. 1996 Sep;60(3):53974.

6. Mahenthiralingam E, Campbell ME, Speert DP. Nonmotility and phagocytic resistance of Pseudomonas aeruginosa isolates from chronically colonized patients with cystic fibrosis. Infect Immun. 1994 Feb;62(2):596605.

7. Winstanley C, O'Brien S, Brockhurst M A. Pseudomonas aeruginosa Evolutionary Adaptation and Diversification in Cystic Fibrosis Chronic Lung Infections. Trends Microbiol. 2016 May;24(5):327-37. DOI: 10.1016/j.tim.2016.01.008

8. Clinical and Laboratory Standards Institute. Performance Standards for Antimicrobial Susceptibility Testing: Twenty-Fifth Informational Supplement
(M100-S25). CLSI, Wayne, PA, USA, 2015.

9. Petermann SR, Doetkott C, Rust L. Elastase deficiency phenotype of Pseudomonas aeruginosa canine otitis externa isolates. Clin Diagn Lab Immunol. 2001 May;8(3):632-6. DOI: 10.1128/CDLI.8.3.632636.2001

10. Burke V, Robinson JO, Richardson CJL, Bundell CS. Longitudinal studies of Virulence Factors of Pseudomonas aeruginosa in Cystic Fibrosis. Pathology 1991 Apr;23(2):145-8. DOI: 10.3109/00313029109060814

11. Høiby N, Frederiksen B, Pressler T. Eradication of early Pseudomonas aeruginosa infection. J Cyst Fibros. 2005 Aug;4(2):49-54. DOI: 10.1016/j.jcf.2005.05.018

12. Islan GA, Tornello PC, Abraham GA, Duran N, Castro GR. Smart lipid nanoparticles containing levofloxacin and DNase for lung delivery. Design and characterization. Colloids SurfB Biointerfaces. 2016 Jul;143(1):16876. DOI: 10.1016/j.colsurfb.2016.03.040

13. Çıragil P, Söyletir G. Alginate, elastase and alkaline protease product, on of Pseudomonas aeruginosa strains isolated from various body sites. Mikrobiyol Bul. 2004 Oct;38(4):341-7.

14. Woods DE, Schaffer MS, Rabin HR, Campbell GD, Sokol PA. Phenotypic comparison of Pseudomonas aeruginosa strains isolated from a variety of clinical sites. J Clin Microbiol. 1986 Aug;24(2):260-4.

15. Hedberg M, Miller JK,Tompkins VN. Elastase Activity of Pseudomonas Aeruginosa Isolates from Hospital Patients. Tech Bull Regist Med Technol. 1969 Oct; 39(10):233-5.

16. Sousa AM, Pereira MO. Pseudomonas aeruginosa diversification during infection development in cystic fibrosis lungs - a review. Pathogens. 2014 Aug;3(3):680703. DOI: 10.3390/pathogens 3030680

17. Amitani R, Wilson R, Rutman A, Read R, Ward C, Burnett D, et al. Effects of Human Neutrophil Elastase and Pseudomonas aeruginosa Proteinases on Human Respiratory Epithelium. Am J Respir Cell Mol Biol. 1991 Jan;4(1):26-32. DOI: 10.1165/ajrcmb/4.1.26

18. Faraji F, Mahzounieh M, Ebrahimi A, Fallah F, Teymournejad O, Lajevardi B. Molecular detection of virulence genes in Pseudomonas aeruginosa isolated from children with Cystic Fibrosis and burn wounds in Iran. Microbial Pathogenesis. 2016 Oct;99:1-4. DOI: 10.1016/j.micpath.2016.07.013

19. Breidenstein EB, de la Fuente-Nú-ez C, Hancock RE. Pseudomonas aeruginosa: all roads lead to resistance. Trends Microbiol. 2011 Aug;19(8):419-26. DOI: 
10.1016/j.tim.2011.04.005

20. Ciofu O. Pseudomonas aeruginosa chromosomal beta-lactamase in patients with cystic fibrosis and chronic lung infection. Mechanism of antibiotic resistance and target of the humoral immune response. APMIS Suppl. 2003;116:1-47.

21. Manno G, Cruciani M, Romano L. Scapolan S, Mentasti M, Lorini R, et al. Antimicrobial use and Pseudomonas aeruginosa susceptibility profile in a cystic fibrosis centre. Int J Antimicrob Agents. 2005 Mar;25(3):1937. DOI: 10.1016/j.ijantimicag.2004.11.009

22. Smith DJ, Ramsay K, Yerkovich ST, Reid DW, Wainwright CE, Grımwood K, et al. Pseudomonas aeruginosa antibiotic resistance in Australian cystic fibrosis centres. Respirology. 2016 Feb;21(2):329-37. DOI: 10.1111/resp. 12714

23. Bosso JA, Mauldin PD, Steed LL. Consequences of
Combining Cystic Fibrosis and Non-Cystic Fibrosis Derived Pseudomonas aeruginosa Antibiotic Susceptibility Results in Hospital Antibiograms. Ann Pharmacother. 2006 Nov;40(11):1946-9. DOI: 10.1345/ aph. $1 \mathrm{H} 377$

24. Çıragil P, Söyletir G, Şener B, Erturan Z. Susceptibilitiy of Pseudomonas aeruginosa Strains Isolated from Cystic Fibrosis and Other Lower Respiratory Tract Infections Against Various Antibiotics. Turk Mikrobiyol Cem Derg. 2002;32(3):197-202.

25. Rao P, McCaughan J, McCalmont M, Goldsmith CE, Hall V, Millar BC, et al. Comparison of antibiotic susceptibility patterns in Pseudomonas aeruginosa isolated from adult patients with cystic fibrosis (CF) with invasive Pseudomonas aeruginosa from non-CF patients. J Cyst Fibros. 2012 Jul;11(4):349-52. DOI: 10.1016/j. jcf.2012.01.002 